\title{
Taxonomy of the family Arenaviridae and the order Bunyavirales: update 2018
}

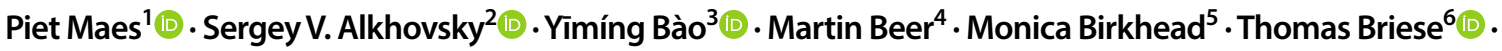
Michael J. Buchmeier ${ }^{7}$. Charles H. Calisher ${ }^{8}$. Rémi N. Charrel ${ }^{9}$. II Ryong Choi ${ }^{10}$. Christopher S. Clegg ${ }^{11}$. Juan Carlos de la Torre ${ }^{12}$. Eric Delwart ${ }^{13,14}$. Joseph L. DeRisi ${ }^{15}$. Patrick L. Di Bello ${ }^{16}$. Francesco Di Serio ${ }^{17}$. Michele Digiaro ${ }^{18}$. Valerian V. Dolja ${ }^{19}$. Christian Drosten ${ }^{20,21,22} \cdot$ Tobiasz Z. Druciarek $^{16}$. Jiang Du ${ }^{23} \cdot$ Hideki Ebihara $^{24}$. Toufic Elbeaino ${ }^{18} \cdot$ Rose C. Gergerich ${ }^{16}$. Amethyst N. Gillis ${ }^{25}$. Jean-Paul J. Gonzalez ${ }^{26}$ - Anne-Lise Haenni ${ }^{27}$.

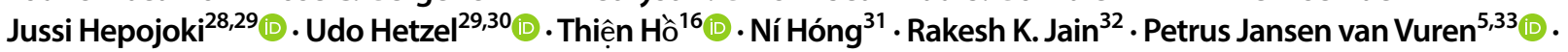
Qi Jin ${ }^{34,35} \cdot$ Miranda Gilda Jonson $^{36} \cdot$ Sandra Junglen ${ }^{20,22} \cdot$ Karen E. Keller $^{37}$ - Alan Kemp ${ }^{5}$ • Anja Kipar ${ }^{29,30}$. Nikola O. Kondov ${ }^{13}$. Eugene V. Koonin ${ }^{38} \cdot$ Richard Kormelink $^{39} \cdot$ Yegor Korzyukov $^{28} \cdot$ Mart Krupovic $^{40}$ (1) Amy J. Lambert ${ }^{41}$. Alma G. Laney ${ }^{42}$ (1) - Matthew LeBreton ${ }^{43} \cdot$ Igor S. Lukashevich ${ }^{44} \cdot$ Marco Marklewitz $^{20,22}$ (1) Wanda Markotter ${ }^{5,33}$ (1) Giovanni P. Martelli ${ }^{45} \cdot$ Robert R. Martin $^{37}$ (1) Nicole Mielke-Ehret ${ }^{46} \cdot$ Hans-Peter Mühlbach $^{46} \cdot$ Beatriz Navarro $^{17}$.

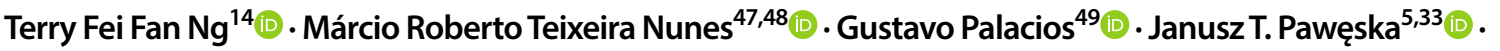
Clarence J. Peters ${ }^{50}$. Alexander Plyusnin ${ }^{28}$. Sheli R. Radoshitzky ${ }^{49}$ - Víctor Romanowski ${ }^{51} \cdot$ Pertteli Salmenperä $^{28,52}$. Maria S. Salvato ${ }^{53} \cdot$ Hélène Sanfaçon ${ }^{54}$. Takahide Sasaya ${ }^{55}$. Connie Schmaljohn ${ }^{49}$. Bradley S. Schneider ${ }^{25}$.

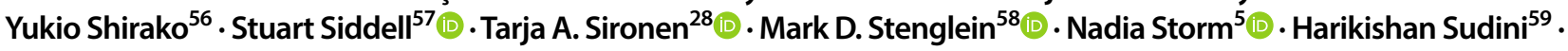
Robert B. Tesh ${ }^{48} \cdot$ loannis E. Tzanetakis ${ }^{16}$ - Mangala Uppala ${ }^{59} \cdot$ Oll Vapalahti $^{28,30,60}$ (D) Nikos Vasilakis ${ }^{48}$ (D) Peter J. Walker ${ }^{61}\left({ }^{10}\right.$. Guópíng Wáng ${ }^{31}$ - Lìpíng Wáng ${ }^{31}$ • Yànxiăng Wáng ${ }^{31}$ - Tàiyún Wèi ${ }^{62}$ - Michael R. Wiley ${ }^{49,63}$.

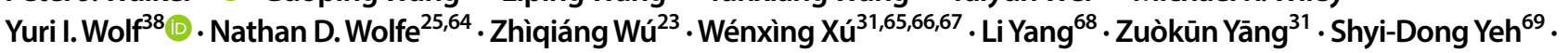
Yǒng-Zhèn Zhāng ${ }^{70}$. Yàzhōu Zhèng ${ }^{31}$ • Xueping Zhou ${ }^{71}$. Chénxī Zhü ${ }^{31}$ • Florian Zirkel ${ }^{21}$ • Jens H. Kuhn ${ }^{72}$

Received: 16 March 2018 / Accepted: 2 April 2018 / Published online: 21 April 2018

(c) This is a U.S. government work and its text is not subject to copyright protection in the United States; however, its text may be subject to foreign copyright protection 2018

Handling Editor: Sead Sabanadzovic.

Michael J. Buchmeier, Rémi N. Charrel, Christopher S. Clegg, Juan Carlos de la Torre, Joseph L. DeRisi, Jean-Paul J. Gonzalez, S. Lukashevich, Clarence J. Peters, Sheli R. Radoshitzky, Víctor Romanowski, Maria S. Salvato and Jens H. Kuhn were the members of the 2014-2017 International Committee on Taxonomy of Viruses (ICTV) Arenaviridae Study Group.

Piet Maes, Sergey V. Alkhovsky, Martin Beer, Thomas Briese, Charles H. Calisher, Rémi N. Charrel, Hideki Ebihara, Rakesh K. Jain, Amy J. Lambert, Márcio Roberto Teixeira Nunes, Alexander Plyusnin, Connie Schmaljohn, Robert B. Tesh, Shyi-Dong Yeh and Jens H. Kuhn were the members of the 2014-2017 ICTV Bunyaviridae Study Group.

Michele Digiaro, Toufic Elbeaino, Giovanni P. Martelli, Nicole Mielke-Ehret and Hans-Peter Mühlbach were the members of the 2014-2017 ICTV Emaravirus Study Group.

Il Ryong Choi, Anne-Lise Haenni, Miranda Gilda Jonson, Takahide Sasaya, Yukio Shirako, Tàiyún Wèi and Xueping Zhou were the members of the 2014-2017 ICTV Tenuivirus Study Group.

Stuart Siddell was the Chair of the 2014-2017 ICTV Animal dsRNA and ssRNA-Viruses Subcommittee.

Hélène Sanfaçon was the Chair of the 2014-2017 ICTV Plant Viruses Subcommittee.

Extended author information available on the last page of the article 


\section{Abstract}

In 2018, the family Arenaviridae was expanded by inclusion of 1 new genus and 5 novel species. At the same time, the recently established order Bunyavirales was expanded by 3 species. This article presents the updated taxonomy of the family Arenaviridae and the order Bunyavirales as now accepted by the International Committee on Taxonomy of Viruses (ICTV) and summarizes additional taxonomic proposals that may affect the order in the near future.

\section{Introduction}

The family Arenaviridae was established in 1976 to accommodate predominantly murid viruses with bisegmented, ambisense single-stranded RNA genomes that form enveloped particles with a "sandy" appearance [7]. Until recently, the family was monogeneric, including the single genus Arenavirus, with a steadily increasing number of species. The taxonomy of the family was substantially amended and emended [22] following the discovery of several distinct arenaviruses in alethinophidian snakes [4, 10, 24]. In particular, the genus Arenavirus was renamed Mammarenavirus, and a second genus, Reptarenavirus, was established in 2014 for several of the newly discovered snake viruses. A non-Linnean binomial species nomenclature was adopted for the entire family Arenaviridae [22] (ICTV TaxoProps [taxonomic proposals] 2014.011a-dV and 2014.012aV). Since then, the genus Mammarenavirus has been extended by 8 species for novel murid viruses discovered in Africa and Asia [3, 8, 11, 15, 21, 25] (TaxoProps 2014.013aV.A.v3. Mammarenavirus_2sp, 2015.001aM, 2016.014aM and 2016.019aM.A.v2.Mammarenavirus_sp).

The order Bunyavirales was established in 2017 to accommodate related viruses with segmented, linear, single-stranded, negative-sense or ambisense RNA genomes distributed among nine families (TaxoProp 2016.030avM). In particular, the then existing family Bunyaviridae was elevated to the rank of order. The three established bunyaviral genera Hantavirus, Nairovirus, and Tospovirus were renamed Orthohantavirus, Orthonairovirus, and Orthotospovirus and included in the newly established families Hantaviridae, Nairoviridae, and Tospoviridae, respectively (TaxoProp 2016.030a-vM). The genus Orthonairovirus was expanded by five species [13] (TaxoProp 2016.026a,bM). The family Peribunyaviridae was created to include the established bunyaviral genus Orthobunyavirus and a new genus, Herbevirus, for bunyaviruses discovered in invertebrates [14, 17] (TaxoProps 2016.024a-dM and 2016.030a-vM). The family Phenuiviridae was created to accommodate the established bunyaviral genus Phlebovirus, the previously "free-floating" plant virus genus Tenuivirus, and two new genera, Goukovirus and Phasivirus, for novel invertebrate bunyaviruses [16] (TaxoProps 2016.022a-dM and 2016.027a-dM). Three new families, Feraviridae, Jonviridae, and Phasmaviridae, were established for newly discovered invertebrate bunyaviruses $[2,14,18]$ (TaxoProps 2016.021a-dM, 2016.025a-dM and 2016.028a-dM).
In addition, the family Fimoviridae was created to accommodate the previously "free-floating" plant virus genus Emaravirus, which was expanded by three species for newly discovered plant viruses [5, 6, 26] (TaxoProps 2016.016aP, 2016.017aP, 2016.018aP, and 2016.030a-vM). Finally, a non-Linnean binomial species nomenclature was adopted for the entire family (TaxoProps 2016.020aM, 2016.023acM, 2016.026a,bM, 2016.029aM.A.v2.Tenuivirus_spren, and 2016.030a-vM).

After the establishment of the order Bunyavirales, the ICTV Study Groups responsible for the taxonomy of Arenaviridae and Bunyavirales assigned unclassified viruses to existing or novel taxa and continued streamlining order nomenclature in collaboration with other virus experts. Here we present the changes to both taxa that were proposed via official TaxoProps at http://www.ictvonline.org/ in 2017 and that were accepted by the ICTV Executive Committee. These changes are official ICTV taxonomy as of March 2018.

\section{Family Arenaviridae}

\section{Taxonomic changes at the family rank}

In 2018 , the family was extended by addition of a novel genus, Hartmanivirus, for Haartman Institute snake virus (HISV) isolated from a captive boa constrictor in Finland [9, 10] (TaxoProp 2017.001 M.A.v1.Hartmanivirus.zip). The genus Mammarenavirus was extended by addition of two novel species for Ryukyo virus (RYKV) and souris virus (SOUV) discovered in mice in China and Cameroon, respectively (TaxoProps 2017.002M.A.v2.Mammarenavirus_sp and 2017.003M.A.v1.Mammarenavirus_sp). Five mammarenavirus species were renamed due to the ICTV decision to disallow diacritic marks in taxon names (TaxoProp 2017.001G.A.v2.43spren): Amaparí mammarenavirus, Junín mammarenavirus, Paraná mammarenavirus, Pichindé mammarenavirus, and Sabiá mammarenavirus were renamed Serra do Navio mammarenavirus, Argentinian mammarenavirus, Paraguayan mammarenavirus, Cali mammarenavirus, and Brazilian mammarenavirus, respectively, whereas the member virus names remained unchanged (TaxoProp 2017.001G.A.v2.43spren). Finally, the three reptarenavirus species names Alethinophid 1 reptarenavirus, Alethinophid 2 reptarenavirus, and Alethinophid 3 reptarenavirus were renamed Golden reptarenavirus, California reptarenavirus, and Rotterdam reptarenavirus. Two new 
reptarenavirus species were created for tavallinen suomalainen mies virus 2 (TSMV-2) and University of Giessen viruses 1-3 (UGV-1-3) discovered in captive boa constrictors; several newly sequenced reptarenaviruses were assigned to existing species [9] (TaxoProp 2017.015M.A.v1. Reptarenavirus_2sp3ren).

\section{Order Bunyavirales}

\section{Taxonomic changes at the order rank}

In 2018, no changes were made at the order rank.

\section{Taxonomic changes at the family rank}

\section{Feraviridae}

In 2018, no changes were made at the family rank.

\section{Fimoviridae}

In 2018 , no changes were made at the family rank.

\section{Hantaviridae}

In 2018, no changes were made at the family rank.

\section{Jonviridae}

In 2018 , no changes were made at the family rank.

\section{Nairoviridae}

The family Nairoviridae was expanded in 2018 by addition of two new species for the long-known but previously unsequenced Artashat virus (ARTSV) and Chim virus (CHIMV), both originally isolated from ticks. In addition, the species Burana orthonairovirus was renamed Tamdy orthonairovirus to better reflect the discovery history of species members, and several newly sequenced nairoviruses were classified into existing species [1] (TaxoProp 2017.008M.A.v1.Orthonairovirus_2sp1ren).

\section{Peribunyaviridae}

The family Peribunyaviridae was expanded in 2018 by addition of a novel species for Wolkberg virus (WBV) discovered in wingless bat flies (Eucampsipoda africana) in South Africa [12] (TaxoProp 2017.007M.A.v1. Orthobunyavirus_sp).

\section{Phasmaviridae}

In 2018 , no changes were made at the family rank.

\section{Phenuiviridae}

In 2018 , no changes were made at the family rank.

\section{Tospoviridae}

In 2018 , no changes were made at the family rank.

\section{Outlook}

The taxonomy of viruses of the family Arenaviridae and the order Mononegavirales remains in flux, and additional important changes are likely forthcoming. Indeed, in 2017, two additional taxonomic proposals that would affect the family Arenaviridae and the order Mononegavirales were debated during the most recent ICTV EC meeting in Singapore. TaxoProp 2017.006M.U.v2.Negarnaviricota proposes the

- establishment of a phylum for negative-sense RNA viruses that is subdivided into two subphyla; and

- establishment of a class including the order Bunyavirales, to be assigned to one of the subphyla.

TaxoProp 2017.012M.U.v2.Bunyavirales_rev proposes

- dissolution of the families Feraviridae, Jonviridae, and Tospoviridae and absorption of their genera into remaining families;

- the creation of three new bunyaviral families for novel invertebrate viruses [19, 20, 23];

- the inclusion of the family Arenaviridae in the order; and

- the creation of 19 new bunyaviral genera. These genera are planned to accommodate novel, mostly invertebrate, viruses $[14,23]$, but some of them are deemed necessary for reclassification of certain hantaviruses and phleboviruses.

These two proposals failed to find unanimous approval at a final ICTV EC vote in fall of 2017 and were deferred to the 2018 ICTV EC meeting, at which a simple majority vote would suffice for approval of the original proposals.

\section{Summary}

Summaries of the current, ICTV-accepted taxonomies of the family Arenaviridae and the order Bunyavirales are presented in Tables 1 and 2, respectively. These tables also 
Table 1 ICTV-accepted taxonomy of the family Arenaviridae as of 2018. Listed are all arenaviruses that have been classified into species

\begin{tabular}{|c|c|c|}
\hline Genus & Species $^{\mathrm{a}}$ & Virus (abbreviation) $^{\mathrm{a}}$ \\
\hline Hartmanivirus & Haartman hartmanivirus & Haartman Institute snake virus (HISV) \\
\hline \multirow[t]{41}{*}{ Mammarenavirus } & Allpahuayo mammarenavirus & Allpahuayo virus (ALLV) \\
\hline & Argentinian mammarenavirus & Junín virus (JUNV) \\
\hline & Bear Canyon mammarenavirus & Bear Canyon virus (BCNV) \\
\hline & Brazilian mammarenavirus & Sabiá virus (SBAV) \\
\hline & Chapare mammarenavirus & Chapare virus (CHAPV) \\
\hline & Cupixi mammarenavirus & Cupixi virus (CUPXV) \\
\hline & Flexal mammarenavirus & Flexal virus (FLEV) \\
\hline & Gairo mammarenavirus & Gairo virus (GAIV) \\
\hline & Guanarito mammarenavirus & Guanarito virus (GTOV) \\
\hline & Ippy mammarenavirus & Ippy virus (IPPYV) \\
\hline & Lassa mammarenavirus & Lassa virus (LASV) \\
\hline & Latino mammarenavirus & Latino virus (LATV) \\
\hline & Loei River mammarenavirus & Loei River virus (LORV) \\
\hline & Lujo mammarenavirus & Lujo virus (LUJV) \\
\hline & Luna mammarenavirus & Luna virus (LUAV) \\
\hline & & Luli virus (LULV) \\
\hline & Lunk mammarenavirus & Lunk virus (LNKV) \\
\hline & Lymphocytic choriomeningitis mammarenavirus ${ }^{\mathrm{b}}$ & lymphocytic choriomeningitis virus (LCMV) \\
\hline & Machupo mammarenavirus & Machupo virus (MACV \\
\hline & Mariental mammarenavirus & Mariental virus (MRLV) \\
\hline & Merino Walk mammarenavirus & Merino Walk virus (MRWV) \\
\hline & Mobala mammarenavirus & mobala virus (MOBV) \\
\hline & Mopeia mammarenavirus & Mopeia virus (MPOV) \\
\hline & & Morogoro virus (MORV) \\
\hline & Okahandja mammarenavirus & Okahandja virus (OKAV) \\
\hline & Oliveros mammarenavirus & Oliveros virus (OLVV) \\
\hline & Paraguayan mammarenavirus & Paraná virus (PRAV) \\
\hline & Cali mammarenavirus & Pichindé virus (PICHV) \\
\hline & Pirital mammarenavirus & Pirital virus (PIRV) \\
\hline & Ryukyu mammarenavirus & Ryukyu virus (RYKV) \\
\hline & Serra do Navio mammarenavirus & Amaparí virus (AMAV) \\
\hline & Solwezi mammarenavirus & Solwezi virus (SOLV) \\
\hline & Souris mammarenavirus & souris virus (SOUV) \\
\hline & Tacaribe mammarenavirus & Tacaribe virus (TCRV) \\
\hline & Tamiami mammarenavirus & Tamiami virus (TMMV) \\
\hline & Wenzhou mammarenavirus & Wēnzhōu virus (WENV) \\
\hline & Whitewater Arroyo mammarenavirus & Big Brushy Tank virus (BBRTV) \\
\hline & & Catarina virus (CTNV) \\
\hline & & Skinner Tank virus (SKTV) \\
\hline & & Tonto Creek virus (TTCV) \\
\hline & & Whitewater Arroyo virus (WWAV) \\
\hline \multirow[t]{8}{*}{ Reptarenavirus } & California reptarenavirus & CAS virus (CASV) \\
\hline & Giessen reptarenavirus & University of Giessen virus 1 (UGV-1) \\
\hline & & University of Giessen virus 2 (UGV-2) \\
\hline & & University of Giessen virus 3 (UGV-3) \\
\hline & Golden reptarenavirus ${ }^{\mathrm{b}}$ & Golden Gate virus (GOGV) \\
\hline & Ordinary reptarenavirus & tavallinen suomalainen mies virus $2(\mathrm{TSMV}-2)$ \\
\hline & Rotterdam reptarenavirus & ROUT virus (ROUTV) \\
\hline & & University of Helsinki virus 1 (UHV-1) \\
\hline
\end{tabular}

${ }^{\text {a }}$ Please note that viruses are real objects that are assigned to concepts that are called taxa. Species, genera, families, and orders are taxa. Taxon names are always italicized and always begin with a capital letter. Virus names, on the other hand, are not italicized and are not capitalized, except if the name or a name component is a proper noun. This column lists the virus names with their correct (lack of) capitalization

${ }^{\mathrm{b}}$ Type species 
Table 2 ICTV-accepted taxonomy of the order Bunyavirales as of 2018. Listed are all bunyaviruses that have been classified into species

\begin{tabular}{|c|c|c|}
\hline Genus & Species $^{\mathrm{a}}$ & Virus (abbreviation) $^{\mathrm{a}}$ \\
\hline \multicolumn{3}{|l|}{ Family Feraviridae } \\
\hline Orthoferavirus & Ferak orthoferavirus ${ }^{\mathrm{b}}$ & ferak virus (FERV) \\
\hline \multicolumn{3}{|l|}{ Family Fimoviridae } \\
\hline \multirow[t]{9}{*}{ Emaravirus } & Actinidia chlorotic ringspot-associated emaravirus & Actinidia chlorotic ringspot-associated virus (AcCRaV) \\
\hline & European mountain ash ringspot-associated emaravirus ${ }^{\mathrm{b}}$ & $\begin{array}{l}\text { European mountain ash ringspot-associated virus } \\
\text { (EMARaV) }\end{array}$ \\
\hline & Fig mosaic emaravirus & fig mosaic virus (FMV) \\
\hline & High Plains wheat mosaic emaravirus & High Plains wheat mosaic virus (HPWMoV) \\
\hline & Pigeonpea sterility mosaic emaravirus 1 & pigeonpea sterility mosaic virus (PPSMV) \\
\hline & Pigeonpea sterility mosaic emaravirus 2 & pigeonpea sterility mosaic virus 2 (PPSMV-2) \\
\hline & Raspberry leaf blotch emaravirus & raspberry leaf blotch virus (RLBV) \\
\hline & Redbud yellow ringspot-associated emaravirus & redbud yellow ringspot-associated virus (RYRaV) \\
\hline & Rose rosette emaravirus & rose rosette virus (RRV) \\
\hline \multicolumn{3}{|c|}{ Family Hantaviridae } \\
\hline \multirow[t]{34}{*}{ Orthohantavirus } & Amga orthohantavirus & Amga virus $(\mathrm{MGAV})^{\mathrm{c}}$ \\
\hline & Andes orthohantavirus & Andes virus (ANDV) \\
\hline & & Castelo dos Sonhos virus (CASV) \\
\hline & & Lechiguanas virus $(\mathrm{LECV}=\mathrm{LECHV})$ \\
\hline & & Orán virus (ORNV) \\
\hline & Asama orthohantavirus & Asama virus (ASAV) \\
\hline & Asikkala orthohantavirus & Asikkala virus (ASIV) \\
\hline & Bayou orthohantavirus & bayou virus (BAYV) \\
\hline & & Catacamas virus (CATV) \\
\hline & Black Creek Canal orthohantavirus & Black Creek Canal virus (BCCV) \\
\hline & Bowe orthohantavirus & Bowé virus (BOWV) \\
\hline & Bruges orthohantavirus & Bruges virus (BRGV) \\
\hline & Cano Delgadito orthohantavirus & Caño Delgadito virus (CADV) \\
\hline & Cao Bang orthohantavirus & Cao Bằng virus (CBNV) \\
\hline & & Liánghé virus (LHEV) \\
\hline & Choclo orthohantavirus & Choclo virus (CHOV) \\
\hline & Dabieshan orthohantavirus & Dàbiéshān virus (DBSV) \\
\hline & Dobrava-Belgrade orthohantavirus & Dobrava virus (DOBV) \\
\hline & & Kurkino virus (KURV) \\
\hline & & Saaremaa virus (SAAV) \\
\hline & & Sochi virus \\
\hline & El Moro Canyon orthohantavirus & Carrizal virus (CARV) \\
\hline & & El Moro Canyon virus (ELMCV) \\
\hline & & Huitzilac virus (HUIV) \\
\hline & Fugong orthohantavirus & Fúgòng virus (FUGV) \\
\hline & Fusong orthohantavirus & Fǔsōng virus (FUSV) \\
\hline & Hantaan orthohantavirus ${ }^{\mathrm{b}}$ & Amur virus (AMRV) \\
\hline & & Hantaan virus (HTNV) \\
\hline & & Soochong virus (SOOV) \\
\hline & Imjin orthohantavirus & Imjin virus (MJNV) \\
\hline & Jeju orthohantavirus & Jeju virus (JJUV) \\
\hline & Kenkeme orthohantavirus & Kenkeme virus (KKMV) \\
\hline & Khabarovsk orthohantavirus & Khabarovsk virus (KHAV) \\
\hline & & Topografov virus (TOPV) \\
\hline
\end{tabular}


Table 2 (continued)

\begin{tabular}{|c|c|c|}
\hline Genus & Species $^{\mathrm{a}}$ & Virus (abbreviation) $^{\mathrm{a}}$ \\
\hline & \multirow[t]{3}{*}{ Laguna Negra orthohantavirus } & Laguna Negra virus (LANV) \\
\hline & & Maripa virus (MARV) \\
\hline & & Río Mamoré virus (RIOMV) \\
\hline & Laibin orthohantavirus & Láibīn virus (LBV) \\
\hline & Longquan orthohantavirus & Lóngquán virus (LQUV) \\
\hline & Luxi orthohantavirus & Lúxī virus (LUXV) \\
\hline & Maporal orthohantavirus & Maporal virus (MAPV) \\
\hline & Montano orthohantavirus & Montaño virus (MTNV) \\
\hline & Necocli orthohantavirus & Necoclí virus (NECV) \\
\hline & Nova orthohantavirus & Nova virus (NVAV) \\
\hline & Oxbow orthohantavirus & Oxbow virus (OXBV) \\
\hline & Prospect Hill orthohantavirus & Prospect Hill virus (PHV) \\
\hline & \multirow[t]{3}{*}{ Puumala orthohantavirus } & Hokkaido virus (HOKV) \\
\hline & & Muju virus (MUJV) \\
\hline & & Puumala virus (PUUV) \\
\hline & Quezon orthohantavirus & Quezon virus (QZNV) \\
\hline & Rockport orthohantavirus & Rockport virus (RKPV) \\
\hline & Sangassou orthohantavirus & Sangassou virus (SANGV) \\
\hline & \multirow[t]{2}{*}{ Seoul orthohantavirus } & gōu virus (GOUV) \\
\hline & & Seoul virus (SEOV) \\
\hline & \multirow[t]{2}{*}{ Sin Nombre orthohantavirus } & New York virus (NYV) ${ }^{\mathrm{d}}$ \\
\hline & & sin nombre virus (SNV) \\
\hline & \multirow[t]{3}{*}{ Thailand orthohantavirus } & Anjozorobe virus \\
\hline & & Serang virus $(\mathrm{SERV})^{\mathrm{e}}$ \\
\hline & & Thailand virus (THAIV) \\
\hline & Thottapalayam orthohantavirus & Thottapalayam virus (TPMV) \\
\hline & \multirow[t]{2}{*}{ Tula orthohantavirus } & Adler virus (ADLV) \\
\hline & & Tula virus (TULV) \\
\hline & Yakeshi orthohantavirus & Yákèshí virus (YKSV) \\
\hline \multicolumn{3}{|l|}{ Family Jonviridae } \\
\hline Orthojonvirus & Jonchet orthojonvirus ${ }^{\mathrm{b}}$ & jonchet virus (JONV) \\
\hline \multicolumn{3}{|l|}{ Family Nairoviridae } \\
\hline \multirow[t]{16}{*}{ Orthonairovirus } & Artashat orthonairovirus & Artashat virus (ARTSV) \\
\hline & Chim orthonairovirus & Chim virus (CHIMV) \\
\hline & Crimean-Congo hemorrhagic fever orthonairovirus & Crimean-Congo hemorrhagic fever virus (CCHFV) \\
\hline & \multirow[t]{4}{*}{ Dera Ghazi Khan orthonairovirus } & Abu Hammad virus $(\mathrm{AHV})^{\mathrm{f}}$ \\
\hline & & Abu Mina virus (AMV) \\
\hline & & Dera Ghazi Khan virus (DGKV) \\
\hline & & Sapphire II virus (SAPV) \\
\hline & \multirow[t]{2}{*}{ Dugbe orthonairovirus ${ }^{\mathrm{b}}$} & Dugbe virus (DUGV) \\
\hline & & kupe virus (KUPEV) \\
\hline & \multirow[t]{2}{*}{ Hazara orthonairovirus } & Hazara virus (HAZV) \\
\hline & & Tofla virus (TFLV) \\
\hline & \multirow[t]{5}{*}{ Hughes orthonairovirus } & Caspiy virus (CASV) \\
\hline & & Farallon virus (FARV) \\
\hline & & Great Saltee virus (GRSV) \\
\hline & & Hughes virus (HUGV) \\
\hline & & Punta Salinas virus (PSV) \\
\hline
\end{tabular}


Table 2 (continued)

\begin{tabular}{|c|c|c|}
\hline Genus & Species $^{\mathrm{a}}$ & Virus (abbreviation) $^{\mathrm{a}}$ \\
\hline & & Raza virus (RAZAV) \\
\hline & & Soldado virus (SOLV) \\
\hline & & Zirqa virus (ZIRV) \\
\hline & Kasokero orthonairovirus & Kasokero virus (KASV = KASOV) \\
\hline & & Leopards Hill virus (LPHV) \\
\hline & & Yogue virus (YOGV) \\
\hline & Keterah orthonairovirus & Gossas virus (GOSV) \\
\hline & & Issyk-kul virus (ISKV) \\
\hline & & Keterah virus (KTRV) ${ }^{\mathrm{g}}$ \\
\hline & & Uzun-Agach virus (UZAV) \\
\hline & Nairobi sheep disease orthonairovirus & Nairobi sheep disease virus (NSDV) ${ }^{\mathrm{h}}$ \\
\hline & Qalyub orthonairovirus & Bandia virus (BDAV) \\
\hline & & Geran virus (GERV) \\
\hline & & Qalyub virus (QYBV) \\
\hline & Sakhalin orthonairovirus & Avalon virus (AVAV) \\
\hline & & Clo Mor virus (CMV = CLMV) \\
\hline & & Sakhalin virus (SAKV) \\
\hline & & Taggert virus (TAGV) \\
\hline & & Tillamook virus (TILLV) \\
\hline & Tamdy orthonairovirus & Burana virus (BURV) \\
\hline & & Huángpí tick virus 1 (HpTV-1) \\
\hline & & Tamdy virus (TAMV) \\
\hline & & Tăchéng tick virus 1 (TcTV-1) \\
\hline & & Wēnzhōu tick virus (WzTV) \\
\hline & Thiafora orthonairovirus & Erve virus (ERVEV) \\
\hline & & Thiafora virus (TFAV) \\
\hline \multicolumn{3}{|c|}{ Family Peribunyaviridae } \\
\hline \multirow[t]{4}{*}{ Herbevirus } & Herbert herbevirus ${ }^{\mathrm{b}}$ & Herbert virus (HEBV) \\
\hline & Kibale herbevirus & Kibale virus (KIBV) \\
\hline & Shuangao insect herbevirus 1 & Shuāngào insect virus 1 (SgIV-1) \\
\hline & Tai herbevirus & Taï virus (TAIV) \\
\hline \multirow[t]{16}{*}{ Orthobunyavirus } & Acara orthobunyavirus & Acará virus (ACAV) \\
\hline & & Moriche virus (MORV) \\
\hline & Akabane orthobunyavirus & Akabane virus (AKAV) \\
\hline & & Sabo virus (SABOV) \\
\hline & & Tinaroo virus (TINV) \\
\hline & & Yaba-7 virus (Y7V) \\
\hline & Alajuela orthobunyavirus & Alajuela virus (ALJV) \\
\hline & & Brus Laguna virus \\
\hline & & San Juan virus (SJV) \\
\hline & Anopheles A orthobunyavirus & Anopheles A virus (ANAV) \\
\hline & & Arumateua virus (ARTV) \\
\hline & & Caraipé virus (CPEV) \\
\hline & & Las Maloyas virus (LMV) \\
\hline & & Lukuni virus (LUKV) \\
\hline & & Trombetas virus (TRMV) \\
\hline & & Tucuruí virus (TUCV) \\
\hline
\end{tabular}


Table 2 (continued)

\begin{tabular}{|c|c|c|}
\hline Genus & Species $^{\mathrm{a}}$ & Virus (abbreviation) $^{\mathrm{a}}$ \\
\hline & \multirow[t]{2}{*}{ Anopheles B orthobunyavirus } & Anopheles B virus (ANBV) \\
\hline & & Boracéia virus (BORV) \\
\hline & \multirow[t]{5}{*}{ Bakau orthobunyavirus } & Bakau virus (BAKV) \\
\hline & & Ketapang virus (KETV) \\
\hline & & Nola virus (NOLAV) \\
\hline & & Tanjong Rabok virus (TRV) \\
\hline & & Telok Forest virus (TFV) \\
\hline & Batama orthobunyavirus & Batama virus (BMAV) \\
\hline & Benevides orthobunyavirus & Benevides virus (BVSV) \\
\hline & \multirow[t]{5}{*}{ Bertioga orthobunyavirus } & Bertioga virus (BERV) \\
\hline & & Cananéia virus (CNAV) \\
\hline & & Guaratuba virus (GTBV) \\
\hline & & Itimirim virus (ITIV) \\
\hline & & Mirim virus (MIRV) \\
\hline & Bimiti orthobunyavirus & bimiti virus (BIMV) \\
\hline & Botambi orthobunyavirus & Botambi virus (BOTV) \\
\hline & \multirow[t]{22}{*}{ Bunyamwera orthobunyavirus ${ }^{\mathrm{b}}$} & Anadyr virus (ANADV) \\
\hline & & Batai virus $(\mathrm{BATV})^{\mathrm{i}}$ \\
\hline & & Birao virus (BIRV) \\
\hline & & Bozo virus (BOZOV) \\
\hline & & Bunyamwera virus (BUNV) \\
\hline & & Cache Valley virus (CVV) \\
\hline & & Fort Sherman virus (FSV) \\
\hline & & Germiston virus (GERV) \\
\hline & & Ilesha virus (ILEV) \\
\hline & & Lokern virus (LOKV) \\
\hline & & Maguari virus (MAGV) \\
\hline & & Mboké virus (MBOV) \\
\hline & & Ngari virus (NRIV) ${ }^{\mathrm{j}}$ \\
\hline & & Northway virus (NORV) \\
\hline & & Playas virus (PLAV) \\
\hline & & Potosi virus (POTV) \\
\hline & & Santa Rosa virus (SARV) \\
\hline & & Shokwe virus (SHOV) \\
\hline & & Stanfield virus (STAV) \\
\hline & & Tensaw virus (TENV) \\
\hline & & Tlacotalpan virus (TLAV) \\
\hline & & Xingu virus (XINV) \\
\hline & \multirow[t]{3}{*}{ Bushbush orthobunyavirus } & Benfica virus (BENV) \\
\hline & & Bushbush virus (BSBV) \\
\hline & & Juan Díaz virus (JDV) \\
\hline & \multirow[t]{2}{*}{ Bwamba orthobunyavirus } & Bwamba virus (BWAV) \\
\hline & & Pongola virus (PGAV) \\
\hline & \multirow[t]{5}{*}{ California encephalitis orthobunyavirus } & Achiote virus (ACHOV) \\
\hline & & California encephalitis virus (CEV) \\
\hline & & infirmatus virus (INFV) \\
\hline & & Inkoo virus (INKV) \\
\hline & & Jamestown Canyon virus (JCV) \\
\hline
\end{tabular}


Table 2 (continued)

\begin{tabular}{|c|c|c|}
\hline Genus & Species $^{\mathrm{a}}$ & Virus (abbreviation) $^{\mathrm{a}}$ \\
\hline & & Jerry Slough virus (JSV) \\
\hline & & Keystone virus (KEYV) \\
\hline & & Khatanga virus (KHATV) ${ }^{\mathrm{k}}$ \\
\hline & & La Crosse virus (LACV) \\
\hline & & Lumbo virus (LUMV) \\
\hline & & Melao virus (MELV) \\
\hline & & Morro Bay virus (MBV) \\
\hline & & San Angelo virus (SAV) \\
\hline & & Serra do Navio virus (SDNV) \\
\hline & & snowshoe hare virus (SSHV) \\
\hline & & South River virus (SORV) \\
\hline & & Ťahyňa virus (TAHV) \\
\hline & & trivittatus virus (TVTV) \\
\hline & Capim orthobunyavirus & Capim virus (CAPV) \\
\hline & Caraparu orthobunyavirus & Apeú virus (APEUV) \\
\hline & & Bruconha virus (BRUV) \\
\hline & & Caraparú virus (CARV) \\
\hline & & El Huayo virus \\
\hline & & Itaya virus (ITYV) \\
\hline & & Ossa virus (OSSAV) \\
\hline & & Vinces virus (VINV) \\
\hline & Catu orthobunyavirus & Catú virus (CATUV) \\
\hline & Estero Real orthobunyavirus & Estero Real virus (ERV) \\
\hline & Gamboa orthobunyavirus & Calchaquí virus (CQIV) \\
\hline & & Gamboa virus (GAMV) \\
\hline & & Pueblo Viejo virus (PVV) \\
\hline & & Soberanía virus \\
\hline & Guajara orthobunyavirus & Guajará virus (GJAV) \\
\hline & Guama orthobunyavirus & Ananindeua virus (ANUV) \\
\hline & & Guamá virus (GMAV) \\
\hline & & Mahogany Hammock virus (MHV) \\
\hline & & Moju virus (MOJUV) \\
\hline & Guaroa orthobunyavirus & Guaroa virus (GROV) \\
\hline & Kaeng Khoi orthobunyavirus & Kaeng Khoi virus (KKV) \\
\hline & Kairi orthobunyavirus & Kairi virus (KRIV) \\
\hline & Koongol orthobunyavirus & koongol virus (KOOV) \\
\hline & & wongal virus (WONV) \\
\hline & Madrid orthobunyavirus & Madrid virus (MADV) \\
\hline & Main Drain orthobunyavirus & Main Drain virus (MDV) \\
\hline & Manzanilla orthobunyavirus & Buttonwillow virus (BUTV) \\
\hline & & Cát Quế virus (CQV) \\
\hline & & Ingwavuma virus (INGV) \\
\hline & & Inini virus (INIV) \\
\hline & & Manzanilla virus (MANV) \\
\hline & & Mermet virus (MERV) \\
\hline & Marituba orthobunyavirus & Gumbo Limbo virus (GLV) \\
\hline & & Marituba virus (MTBV) \\
\hline & & Murutucú virus (MURV) \\
\hline & & Nepuyo virus (NEPV) \\
\hline
\end{tabular}


Table 2 (continued)

\begin{tabular}{|c|c|c|}
\hline Genus & Species $^{\mathrm{a}}$ & Virus (abbreviation) $^{\mathrm{a}}$ \\
\hline & \multirow{4}{*}{ Minatitlan orthobunyavirus } & Restan virus (RESV) \\
\hline & & Zungarococha virus (ZUNV) \\
\hline & & Minatitlán virus (MNTV) \\
\hline & & Palestina virus (PLSV) \\
\hline & \multirow[t]{2}{*}{ MPoko orthobunyavirus } & M'Poko virus (MPOV) \\
\hline & & Yaba-1 virus (Y1V) \\
\hline & \multirow[t]{2}{*}{ Nyando orthobunyavirus } & Nyando virus (NDV) \\
\hline & & Eretmapodites virus (ERETV) \\
\hline & \multirow[t]{4}{*}{ Olifantsvlei orthobunyavirus } & Bobia virus (BIAV) \\
\hline & & Dabakala virus (DABV) \\
\hline & & Olifantsvlei virus (OLIV) \\
\hline & & Oubi virus (OUBIV) \\
\hline & \multirow[t]{2}{*}{ Oriboca orthobunyavirus } & Itaquí virus (ITQV) \\
\hline & & Oriboca virus (ORIV) \\
\hline & \multirow[t]{8}{*}{ Oropouche orthobunyavirus } & Facey’s paddock virus (FPV) \\
\hline & & Iquitos virus (IQTV) ${ }^{\mathrm{d}}$ \\
\hline & & Madre de Dios virus (MDDV) \\
\hline & & Oropouche virus (OROV) \\
\hline & & Perdões virus (PDEV) \\
\hline & & Pintupo virus (PINTV) \\
\hline & & Utinga virus (UTIV) \\
\hline & & Utivé virus (UVV = UTVEV) \\
\hline & \multirow[t]{5}{*}{ Patois orthobunyavirus } & Abras virus (ABRV) \\
\hline & & Babahoya virus (BABV) \\
\hline & & Pahayokee virus (PAHV) \\
\hline & & Patois virus (PATV) \\
\hline & & Shark River virus (SRV) \\
\hline & \multirow[t]{2}{*}{ Sathuperi orthobunyavirus } & Douglas virus (DOUV) \\
\hline & & Sathuperi virus (SATV) \\
\hline & \multirow[t]{3}{*}{ Shamonda orthobunyavirus } & Peaton virus (PEAV) \\
\hline & & Sango virus (SANV) \\
\hline & & Shamonda virus (SHAV) \\
\hline & \multirow[t]{3}{*}{ Shuni orthobunyavirus } & Aino virus (AINOV) \\
\hline & & Kaikalur virus (KAIV) \\
\hline & & Shuni virus (SHUV) \\
\hline & \multirow[t]{2}{*}{ Simbu orthobunyavirus } & Simbu virus (SIMV) \\
\hline & & Oya virus (OYAV) \\
\hline & \multirow[t]{4}{*}{ Tacaiuma orthobunyavirus } & Tacaiuma virus (TCMV) \\
\hline & & CoAr 1071 virus (CA1071V) \\
\hline & & CoAr 3627 virus (CA3626V) \\
\hline & & Virgin River virus (VRV) \\
\hline & \multirow[t]{5}{*}{ Tete orthobunyavirus } & Bahig virus (BAHV) \\
\hline & & Matruh virus (MTRV) \\
\hline & & Tete virus (TETEV) \\
\hline & & Tsuruse virus (TSUV) \\
\hline & & Weldona virus (WELV) \\
\hline & Thimiri orthobunyavirus & Thimiri virus (THIV) \\
\hline & Timboteua orthobunyavirus & Timboteua virus (TBTV) \\
\hline
\end{tabular}


Table 2 (continued)

\begin{tabular}{|c|c|c|}
\hline Genus & Species $^{\mathrm{a}}$ & Virus (abbreviation) $^{\mathrm{a}}$ \\
\hline & Turlock orthobunyavirus & Lednice virus (LEDV) \\
\hline & & Turlock virus (TURV) \\
\hline & & Umbre virus (UMBV) \\
\hline & Wolkberg orthobunyavirus & Wolkberg virus (WBV) \\
\hline & Wyeomyia orthobunyavirus & Anhembi virus (AMBV) \\
\hline & & BeAr 328208 virus (BAV) \\
\hline & & Cachoeira Porteira virus (CPOV) \\
\hline & & Iaco virus (IACOV) \\
\hline & & Macauã virus (MCAV) \\
\hline & & Rio Pracupi virus \\
\hline & & Sororoca virus (SORV) \\
\hline & & Taiassui virus (TAIAV) \\
\hline & & Tucunduba virus (TUCV) \\
\hline & & Wyeomyia virus (WYOV) \\
\hline & Zegla orthobunyavirus & Zegla virus (ZEGV) \\
\hline \multicolumn{3}{|l|}{ Family Phasmaviridae } \\
\hline \multirow[t]{6}{*}{ Orthophasmavirus } & Kigluaik phantom orthophasmavirus ${ }^{\mathrm{b}}$ & Kigluaik phantom virus (KIGV) \\
\hline & Nome phantom orthophasmavirus & Nome phantom virus (NOMV) \\
\hline & Shuangao insect orthophasmavirus 2 & Shuāngào insect virus 2 (SgIV-2) \\
\hline & Wuchang cockroach orthophasmavirus 1 & Wǔchāng cockroach virus 1 (WcCV-1) \\
\hline & Wuhan mosquito orthophasmavirus 1 & Wǔhàn mosquito virus 1 (WhMV-1) \\
\hline & Wuhan mosquito orthophasmavirus 2 & Wǔhàn mosquito virus 2 (WhMV-2) \\
\hline \multicolumn{3}{|l|}{ Family Phenuiviridae } \\
\hline \multirow[t]{3}{*}{ Goukovirus } & Cumuto goukovirus & Cumuto virus (CUMV) \\
\hline & Gouleako goukovirus ${ }^{\mathrm{b}}$ & Gouléako virus (GOLV) \\
\hline & Yichang insect goukovirus & Yíchāng insect virus (YcIV) \\
\hline \multirow[t]{4}{*}{ Phasivirus } & Badu phasivirus ${ }^{\mathrm{b}}$ & Badu virus (BADUV) \\
\hline & Phasi Charoen-like phasivirus & Phasi Chaeron-like virus (PCLV) \\
\hline & Wuhan fly phasivirus & Wǔhàn fly virus 1 (WhFV-1) \\
\hline & Wutai mosquito phasivirus & Wǔtái mosquito virus (WtMV) \\
\hline \multirow[t]{20}{*}{ Phlebovirus } & Bujaru phlebovirus & Bujaru virus (BUJV) \\
\hline & & Munguba virus (MUNV) \\
\hline & Candiru phlebovirus & Alenquer virus (ALEV) \\
\hline & & Ariquemes virus (ARQV) \\
\hline & & Candiru virus (CDUV) \\
\hline & & Itaituba virus (ITAV) \\
\hline & & Jacundá virus (JCNV) \\
\hline & & Maldonado virus (MLOV) \\
\hline & & Morumbi virus (MR(M)BV) \\
\hline & & Mucura virus (MCRV/MRAV) \\
\hline & & Nique virus (NIQV) \\
\hline & & Oriximiná virus (ORXV) \\
\hline & & Serra Norte virus (SRNV) \\
\hline & & Turuna virus (TUAV) \\
\hline & Chilibre phlebovirus & Cacao virus (CACV) \\
\hline & & Chilibre virus (CHIV) \\
\hline & Frijoles phlebovirus & Frijoles virus (FRIV) \\
\hline & & Joá virus (JOAV) \\
\hline & Punta Toro phlebovirus & Buenaventura virus (BUEV) \\
\hline & & Campana virus (CMAV) \\
\hline
\end{tabular}


Table 2 (continued)

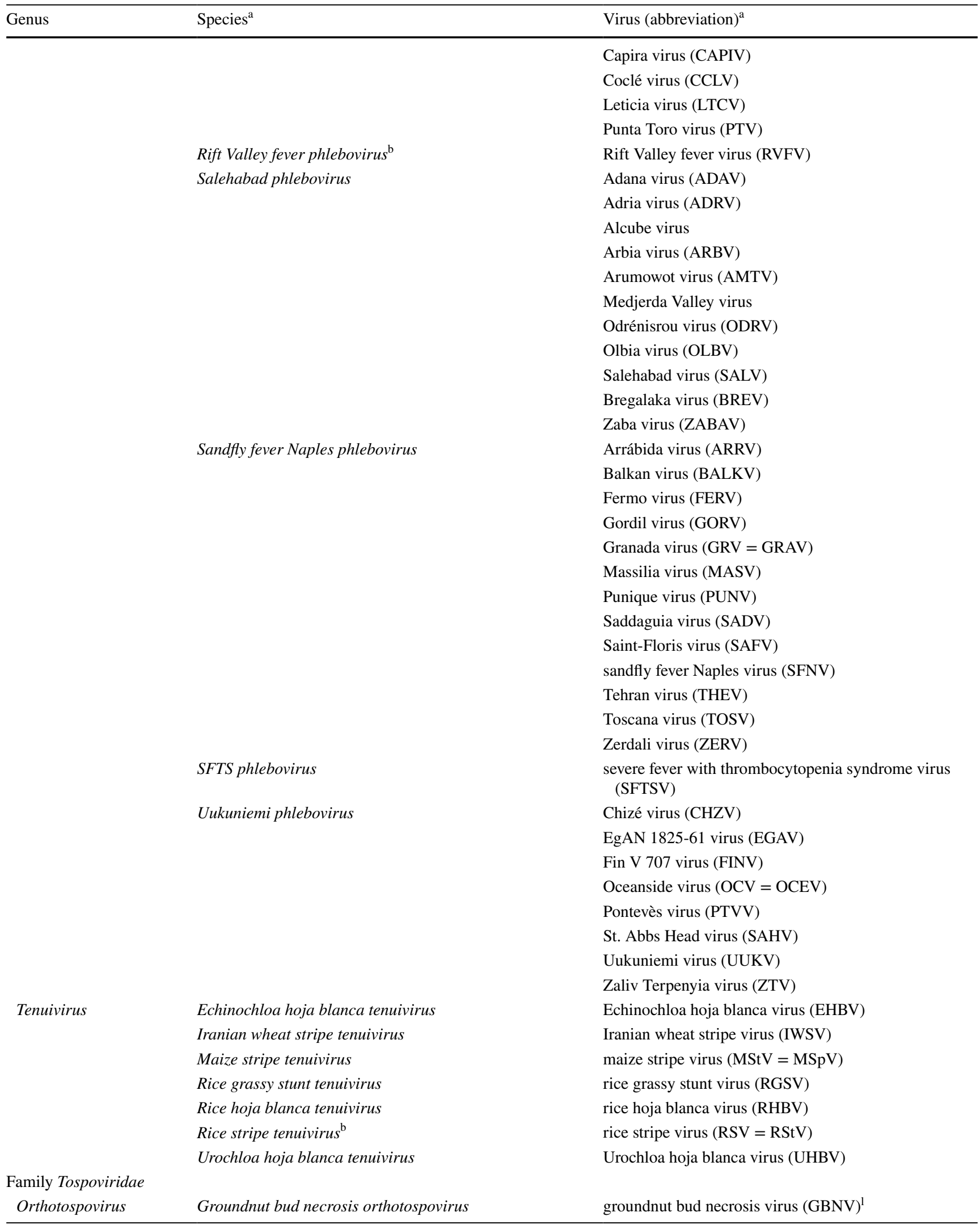


Table 2 (continued)

\begin{tabular}{|c|c|c|}
\hline Genus & Species $^{\mathrm{a}}$ & Virus (abbreviation) $^{\mathrm{a}}$ \\
\hline & Groundnut ringspot orthotospovirus & groundnut ringspot virus (GRSV) \\
\hline & Groundnut yellow spot orthotospovirus & groundnut yellow spot virus $(\mathrm{GYSV})^{\mathrm{m}}$ \\
\hline & Impatiens necrotic spot orthotospovirus & impatiens necrotic spot virus (INSV) \\
\hline & Iris yellow spot orthotospovirus & iris yellow spot virus (IYSV) \\
\hline & Polygonum ringspot orthotospovirus & Polygonum ringspot virus (PolRSV) \\
\hline & Tomato chlorotic spot orthotospovirus & tomato chlorotic spot virus (TCSV) \\
\hline & Tomato spotted wilt orthotospovirus ${ }^{\mathrm{b}}$ & tomato spotted wilt virus (TSWV) \\
\hline & Watermelon bud necrosis orthotospovirus & watermelon bud necrosis virus (WBNV) \\
\hline & Watermelon silver mottle orthotospovirus & watermelon silver mottle virus (WSMoV) \\
\hline & Zucchini lethal chlorosis orthotospovirus & zucchini lethal chlorosis virus (ZLCV) \\
\hline
\end{tabular}

aPlease note that viruses are real objects that are assigned to concepts that are called taxa. Species, genera, families, and orders are taxa. Taxon names are always italicized and always begin with a capital letter. Virus names, on the other hand, are not italicized and are not capitalized, except if the name or a name component is a proper noun. This column lists the virus names with their correct (lack of) capitalization. Lists of viruses within a given species are provisional at this point and will likely be amended in the near future

${ }^{\mathrm{b}}$ Type species

${ }^{\mathrm{c} S y n o n y m: ~ A r t y b a s h ~ v i r u s ~(A R T V) ~}$

${ }^{\mathrm{d}}$ Synonym: New York 1 virus (NY-1V)

${ }^{\text {e}}$ Synonym: Jurong virus

${ }^{\mathrm{f}}$ Includes the strain previously referred to as Tunis virus (TUNV)

${ }^{\mathrm{g}}$ Includes the strain previously referred to as soft tick bunyavirus (STBV)

${ }^{\mathrm{h}}$ Includes the strain previously referred to as Ganjam virus (GANV)

${ }^{i}$ Synonyms: Čalovo virus (CVOV), Chittoor virus (CHITV), Olkya virus, Olyka virus, UgMP-6830 virus

${ }^{\mathrm{j}}$ Includes the strain previously referred to as Garissa virus

${ }^{\mathrm{k}}$ Also mistakenly referred to in the literature as Chantanga virus (CHATV) and Chatanga virus (CHATV)

${ }^{1}$ Synonym: peanut bud necrosis virus (PDNV)

${ }^{\mathrm{m}}$ Synonym: peanut yellow spot virus (PYSV)

include corrections and updates in virus name or abbreviation spelling.

Acknowledgments We thank Laura Bollinger (NIH/NIAID Integrated Research Facility at Fort Detrick, Frederick, MD, USA) and F. Murilo Zerbini (BIOAGRO, Universidade Federal de Viçosa, Viçosa, Brazil) for critically editing the manuscript.

Funding This work was supported in part through Battelle Memorial Institute's prime contract with the US National Institute of Allergy and Infectious Diseases (NIAID) under Contract No. HHSN272200700016I (J.H.K.). This work was also funded in part by National Institutes of Health (NIH) contract HHSN272201000040I/HHSN27200004/D04 and Grant R24AI120942 (N.V., R.B.T.), intramural funds of the US Department of Health and Human Services to the US National Library of Medicine (E.V.K. and Y.I.W.), and the 100-Talent Program of the Chinese Academy of Sciences, the National Key R\&D Program of China (2016YFE0206600) and the International Union of Biological Sciences (Y.B.)

\section{Compliance with ethical standards}

Ethical approval The views and conclusions contained in this document are those of the authors and should not be interpreted as necessarily representing the official policies, either expressed or implied, of the US Department of the Army, the US Department of Defense, the US Department of Health and Human Services, or of the institutions and companies affiliated with the authors. In no event shall any of these entities have any responsibility or liability for any use, misuse, inability to use, or reliance upon the information contained herein. The US departments do not endorse any products or commercial services mentioned in this publication.

Conflict of interest The authors declare no conflicts of interest.

Ethical approval This article does not contain any studies with human participants or animals performed by any of the authors.

\section{References}

1. Alkhovsky SV, Lvov DK, Shchetinin AM, Deriabin PG, Shchelkanov MY, Aristova VA, Morozova TN, Gitelman AK, Palacios GF, Kuhn JH (2017) Complete genome coding sequences of Artashat, Burana, Caspiy, Chim, Geran, Tamdy, and UzunAgach viruses (Bunyavirales: Nairoviridae: Orthonairovirus). Genome Announc 5:e01098-01017

2. Ballinger MJ, Bruenn JA, Hay J, Czechowski D, Taylor DJ (2014) Discovery and evolution of bunyavirids in arctic 
phantom midges and ancient bunyavirid-like sequences in insect genomes. J Virol 88:8783-8794

3. Blasdell KR, Duong V, Eloit M, Chretien F, Ly S, Hul V, Deubel V, Morand S, Buchy P (2016) Evidence of human infection by a new mammarena virus endemic to Southeastern Asia. Elife 5:e13135

4. Bodewes R, Kik MJL, Raj VS, Schapendonk CME, Haagmans BL, Smits SL, Osterhaus ADME (2013) Detection of novel divergent arenaviruses in boid snakes with inclusion body disease in The Netherlands. J Gen Virol 94:1206-1210

5. Di Bello PL, Laney AG, Druciarek T, Ho T, Gergerich RC, Keller KE, Martin RR, Tzanetakis IE (2016) A novel emaravirus is associated with redbud yellow ringspot disease. Virus Res 222:41-47

6. Elbeaino T, Digiaro M, Uppala M, Sudini H (2015) Deep sequencing of dsRNAs recovered from mosaic-diseased pigeonpea reveals the presence of a novel emaravirus: pigeonpea sterility mosaic virus 2. Arch Virol 160:2019-2029

7. Fenner F (1976) Classification and nomenclature of viruses. Second report of the International Committee on Taxonomy of Viruses. Intervirology 7:1-115

8. Gryseels S, Rieger T, Oestereich L, Cuypers B, Borremans B, Makundi R, Leirs H, Günther S, Goüy de Bellocq J (2015) Gairo virus, a novel arenavirus of the widespread Mastomys natalensis: genetically divergent, but ecologically similar to Lassa and Morogoro viruses. Virology 476:249-256

9. Hepojoki J, Salmenperä P, Sironen T, Hetzel U, Korzyukov Y, Kipar A, Vapalahti O (2015) Arenavirus coinfections are common in snakes with boid inclusion body disease. J Virol 89:8657-8660

10. Hetzel U, Sironen T, Laurinmäki P, Liljeroos L, Patjas A, Henttonen H, Vaheri A, Artelt A, Kipar A, Butcher SJ, Vapalahti O, Hepojoki J (2013) Isolation, identification, and characterization of novel arenaviruses, the etiological agents of boid inclusion body disease. J Virol 87:10918-10935

11. Ishii A, Thomas $\mathrm{Y}$, Moonga L, Nakamura I, Ohnuma A, Hang'ombe BM, Takada A, Mweene AS, Sawa H (2012) Molecular surveillance and phylogenetic analysis of Old World arenaviruses in Zambia. J Gen Virol 93:2247-2251

12. Jansen van Vuren P, Wiley MR, Palacios G, Storm N, Markotter W, Birkhead M, Kemp A, Paweska JT (2017) Isolation of a novel orthobunyavirus from bat flies (Eucampsipoda africana). J Gen Virol 98:935-945

13. Kuhn JH, Wiley MR, Rodriguez SE, Bào Y, Prieto K, Travassos da Rosa APA, Guzman H, Savji N, Ladner JT, Tesh RB, Wada J, Jahrling PB, Bente DA, Palacios G (2016) Genomic characterization of the genus Nairovirus (family Bunyaviridae). Viruses 8:164

14. Li C-X, Shi M, Tian J-H, Lin X-D, Kang Y-J, Chen L-J, Qin X-C, Xu J, Holmes EC, Zhang Y-Z (2015) Unprecedented genomic diversity of RNA viruses in arthropods reveals the ancestry of negative-sense RNA viruses. Elife 4:e05378

15. Li K, Lin X-D, Wang W, Shi M, Guo W-P, Zhang X-H, Xing J-G, He J-R, Wang K, Li M-H, Cao J-H, Jiang M-L, Holmes EC,
Zhang Y-Z (2015) Isolation and characterization of a novel arenavirus harbored by rodents and shrews in Zhejiang province, China. Virology 476:37-42

16. Marklewitz M, Handrick S, Grasse W, Kurth A, Lukashev A, Drosten C, Ellerbrok H, Leendertz FH, Pauli G, Junglen S (2011) Gouléako virus isolated from West African mosquitoes constitutes a proposed novel genus in the family Bunyaviridae. J Virol 85:9227-9234

17. Marklewitz M, Zirkel F, Rwego IB, Heidemann H, Trippner P, Kurth A, Kallies R, Briese T, Lipkin WI, Drosten C, Gillespie TR, Junglen S (2013) Discovery of a unique novel clade of mosquitoassociated bunyaviruses. J Virol 87:12850-12865

18. Marklewitz M, Zirkel F, Kurth A, Drosten C, Junglen S (2015) Evolutionary and phenotypic analysis of live virus isolates suggests arthropod origin of a pathogenic RNA virus family. Proc Natl Acad Sci USA 112:7536-7541

19. Marzano S-YL, Domier LL (2016) Novel mycoviruses discovered from metatranscriptomics survey of soybean phyllosphere phytobiomes. Virus Res 213:332-342

20. Marzano S-YL, Nelson BD, Ajayi-Oyetunde O, Bradley CA Hughes TJ, Hartman GL, Eastburn DM, Domier LL (2016) Identification of diverse mycoviruses through metatranscriptomics characterization of the viromes of five major fungal plant pathogens. J Virol 90:6846-6863

21. Palacios G, Savji N, Hui J, Travassos da Rosa A, Popov V, Briese T, Tesh R, Lipkin WI (2010) Genomic and phylogenetic characterization of Merino Walk virus, a novel arenavirus isolated in South Africa. J Gen Virol 91:1315-1324

22. Radoshitzky SR, Bào Y, Buchmeier MJ, Charrel RN, Clawson AN, Clegg CS, DeRisi JL, Emonet S, Gonzalez J-P, Kuhn JH, Lukashevich IS, Peters CJ, Romanowski V, Salvato MS, Stenglein MD, de la Torre JC (2015) Past, present, and future of arenavirus taxonomy. Arch Virol 160:1851-1874

23. Shi M, Lin X-D, Tian J-H, Chen L-J, Chen X, Li C-X, Qin X-C, Li J, Cao J-P, Eden J-S, Buchmann J, Wang W, Xu J, Holmes EC, Zhang Y-Z (2016) Redefining the invertebrate RNA virosphere. Nature 540:539-543

24. Stenglein MD, Sanders C, Kistler AL, Ruby JG, Franco JY, Reavill DR, Dunker F, DeRisi JL (2012) Identification, characterization, and in vitro culture of highly divergent arenaviruses from boa constrictors and annulated tree boas: candidate etiological agents for snake inclusion body disease. MBio 3:e0180-00112

25. Witkowski PT, Kallies R, Hoveka J, Auste B, Ithete NL, Šoltys K, Szemes T, Drosten C, Preiser W, Klempa B, Mfune JKE, Kruger DH (2015) Novel arenavirus isolates from Namaqua rock mice, Namibia, Southern Africa. Emerg Infect Dis 21:1213-1216

26. Zheng Y, Navarro B, Wang G, Wang Y, Yang Z, Xu W, Zhu C, Wang L, Di Serio F, Hong N (2017) Actinidia chlorotic ringspotassociated virus: a novel emaravirus infecting kiwifruit plants. Mol Plant Pathol 18:569-581

\section{Affiliations}

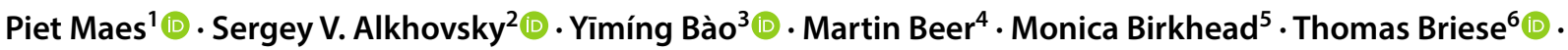
Michael J. Buchmeier ${ }^{7}$. . Charles H. Calisher ${ }^{8} \cdot$ Rémi N. Charrel $^{9}$. II Ryong Choi ${ }^{10}$. Christopher S. Clegg ${ }^{11}$. Juan Carlos de la Torre ${ }^{12}$. Eric Delwart ${ }^{13,14}$. Joseph L. DeRisi ${ }^{15}$. Patrick L. Di Bello ${ }^{16}$. Francesco Di Serio ${ }^{17}$. Michele Digiaro ${ }^{18}$. Valerian V. Dolja ${ }^{19}$. Christian Drosten ${ }^{20,21,22} \cdot$ Tobiasz Z. Druciarek $^{16}$. Jiang Du ${ }^{23}$. Hideki Ebihara ${ }^{24}$. Toufic Elbeaino ${ }^{18} \cdot$ Rose C. Gergerich ${ }^{16}$. Amethyst N. Gillis ${ }^{25}$. Jean-Paul J. Gonzalez ${ }^{26}$ (1) . Anne-Lise Haenni ${ }^{27}$. Jussi Hepojoki ${ }^{28,29}$. Udo Hetzel ${ }^{29,30}$ - Thiện Hồ ${ }^{16}$ (1) Ní Hóng ${ }^{31} \cdot$ Rakesh K. Jain $^{32}$. 
Petrus Jansen van Vuren ${ }^{5,33} \oplus$ - Qi Jin ${ }^{34,35} \cdot$ Miranda Gilda Jonson ${ }^{36} \cdot$ Sandra Junglen $^{20,22} \cdot$ Karen E. Keller $^{37}$. Alan Kemp ${ }^{5}$ - Anja Kipar ${ }^{29,30}$ - Nikola O. Kondov ${ }^{13} \cdot$ Eugene V. Koonin $^{38} \cdot$ Richard Kormelink $^{39}$. Yegor Korzyukov ${ }^{28} \cdot$ Mart Krupovic $^{40}$ (1) - Amy J. Lambert ${ }^{41}$. Alma G. Laney ${ }^{42}$ (i) $\cdot$ Matthew LeBreton $^{43}$.

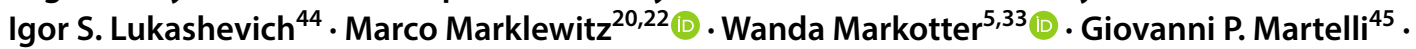
Robert R. Martin ${ }^{37}$ (D) Nicole Mielke-Ehret ${ }^{46}$. Hans-Peter Mühlbach ${ }^{46}$. Beatriz Navarro ${ }^{17}$. Terry Fei Fan $\mathrm{Ng}^{14}$ (D) Márcio Roberto Teixeira Nunes ${ }^{47,48}$ (1) . Gustavo Palacios ${ }^{49}$ (1) . Janusz T. Pawęska ${ }^{5,33}$ (1) . Clarence J. Peters ${ }^{50}$. Alexander Plyusnin ${ }^{28}$. Sheli R. Radoshitzky ${ }^{49}$ - Víctor Romanowski ${ }^{51}$ • Pertteli Salmenperä ${ }^{28,52} \cdot$ Maria S. Salvato $^{53}$. Hélène Sanfaçon ${ }^{54}$ (1) Takahide Sasaya ${ }^{55}$ - Connie Schmaljohn ${ }^{49} \cdot$ Bradley S. Schneider $^{25} \cdot$ Yukio Shirako $^{56}$.

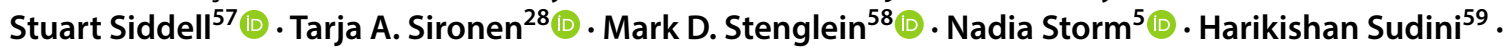

Robert B. Tesh ${ }^{48} \cdot$ loannis E. Tzanetakis ${ }^{16}$ - Mangala Uppala ${ }^{59} \cdot$ Olli Vapalahti $^{28,30,60}$ - Nikos Vasilakis ${ }^{48}$ (1) Peter J. Walker ${ }^{61}$ (1) - Guópíng Wáng ${ }^{31}$ • Lìpíng Wáng ${ }^{31}$ - Yànxiăng Wáng ${ }^{31}$ · Tàiyún Wèi ${ }^{62}$. Michael R. Wiley ${ }^{49,63}$.

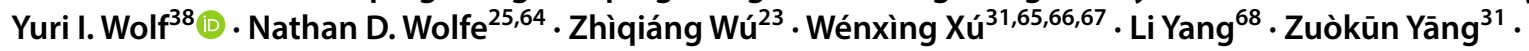
Shyi-Dong Yeh ${ }^{69}$. Yǒng-Zhèn Zhāng ${ }^{70}$. Yàzhōu Zhèng ${ }^{31}$. Xueping Zhou ${ }^{71}$. Chénxī Zhü ${ }^{31}$. Florian Zirkel ${ }^{21}$. Jens H. Kuhn ${ }^{72}$ (I)

$\checkmark$ Jens H. Kuhn kuhnjens@mail.nih.gov

1 Zoonotic Infectious Diseases Unit, KU Leuven, Leuven, Belgium

2 D. I. Ivanovsky Institute of Virology, N. F. Gamaleya Federal Research Center for Epidemiology and Microbiology, Ministry of Health of the Russian Federation, Moscow, Russia

3 Beijing Institute of Genomics, Chinese Academy of Sciences, Beijing, China

4 Institute of Diagnostic Virology, Friedrich-Loeffler-Institut, Greifswald-Insel Riems, Germany

5 Centre for Emerging Zoonotic and Parasitic Diseases, National Institute for Communicable Diseases, National Health Laboratory Service, Sandringham, South Africa

6 Department of Epidemiology, Center for Infection and Immunity, Mailman School of Public Health, Columbia University, New York, New York, USA

7 Department of Molecular Biology and Biochemistry, University of California, Irvine, CA, USA

8 Arthropod-Borne and Infectious Diseases Laboratory, College of Veterinary Medicine and Biomedical Sciences, Colorado State University, Fort Collins, CO, USA

9 Unité des Virus Emergents, Aix-Marseille University, IRD 190, Inserm 1207, IHU Méditerranée Infection, Marseille, France

10 Plant Breeding Genetics and Biotechnology Division, International Rice Research Institute, Los Baños, Philippines

11 Les Mandinaux, Le Grand Madieu, France

12 Department of Immunology and Microbial Science, IMM-6, The Scripps Research Institute, La Jolla, CA, USA

13 Blood Systems Research Institute, San Francisco, CA, USA

14 Department of Laboratory Medicine, University of California, San Francisco, San Francisco, CA, USA

15 Departments of Medicine, Biochemistry and Biophysics, and Microbiology, University of California, San Francisco, CA, USA
16 Division of Agriculture, Department of Plant Pathology, University of Arkansas System, Fayetteville, AR, USA

17 Istituto per la Protezione Sostenibile delle Piante, Consiglio Nazionale delle Ricerche, Bari, Italy

18 Istituto Agronomico Mediterraneo, Valenzano, Italy

19 Department of Botany and Plant Pathology, Center for Genome Research and Biocomputing, Oregon State University, Corvallis, OR, USA

20 Institute of Virology, Charité, Universitätsmedizin Berlin, Corporate Member of Free University Berlin, Berlin Institute of Health, Humboldt-University Berlin, Berlin, Germany

21 Institute of Virology, University of Bonn Medical Centre, Bonn, Germany

22 German Centre for Infection Research, Braunschweig, Germany

23 Institute of Pathogen Biology, Chinese Academy of Medical Sciences, Beijing, China

24 Department of Molecular Medicine, Mayo Clinic, Rochester, MN, USA

25 Metabiota, San Francisco, CA, USA

26 Center of Excellence for Emerging and Zoonotic Animal Disease, Kansas State University, Manhattan, KS, USA

27 Institut Jacques Monod, CNRS, Université Paris-Diderot, Paris, France

28 Department of Virology, University of Helsinki, Medicum, Helsinki, Finland

29 Vetsuisse Faculty, Institute of Veterinary Pathology, University of Zurich, Zurich, Switzerland

30 Department of Veterinary Biosciences, Faculty of Veterinary Medicine, University of Helsinki, Helsinki, Finland

31 State Key Laboratory of Agromicrobiology, Huazhong Agricultural University, Wuhan, Hubei, China

32 Division of Plant Pathology, Indian Agricultural Research Institute, New Delhi, India

33 Department of Medical Virology, Faculty of Health Sciences, Centre for Viral Zoonoses, University of Pretoria, Pretoria, South Africa 
34 MOH Key Laboratory of Systems Biology of Pathogens, Institute of Pathogen Biology, Peking Union Medical College, Chinese Academy of Medical Sciences, Beijing, China

35 Collaborative Innovation Center for Diagnosis and Treatment of Infectious Diseases, Beijing, China

36 Department of Agricultural Biotechnology, Center for Fungal Pathogenesis, College of Agriculture and Life Sciences, Seoul National University, Seoul, Korea

37 United States Department of Agriculture, Agricultural Research Service, Horticultural Crops Research Laboratory, Corvallis, OR, USA

38 National Center for Biotechnology Information, National Library of Medicine, National Institutes of Health, Bethesda, MD, USA

39 Laboratory of Virology, Department of Plant Sciences, Wageningen University, Wageningen, The Netherlands

40 Department of Microbiology, Institut Pasteur, Paris, France

41 Division of Vector-Borne Diseases, National Center for Emerging and Zoonotic Infectious Diseases, Centers for Disease Control and Prevention, Fort Collins, CO, USA

42 Department of Entomology and Plant Pathology, North Carolina State University, Raleigh, NC, USA

43 Mosaic, Yaoundé, Cameroon

44 Department of Pharmacology and Toxicology, School of Medicine, Center for Predictive Medicine for Biodefense and Emerging Infectious Diseases, University of Louisville, Louisville, KY, USA

45 Department of Plant, Soil and Food Sciences, University "Aldo Moro", Bari, Italy

46 Biocentre Klein Flottbek, University of Hamburg, Hamburg, Germany

47 Evandro Chagas Institute, Ministry of Health, Pará, Brazil

48 Department of Pathology, Center for Biodefense and Emerging Infectious Diseases, University of Texas Medical Branch, Galveston, TX, USA

49 United States Army Medical Research Institute of Infectious Diseases, Fort Detrick, Frederick, MD, USA

50 Galveston National Laboratory, University of Texas Medical Branch, Galveston, TX, USA

51 Instituto de Biotecnología y Biología Molecular, Centro Cientifico Technológico-La Plata, Consejo Nacional de Investigaciones Científicas y Técnicas, Universidad Nacional de La Plata, La Plata, Argentina

52 Blueprint Genetics, Helsinki, Finland

53 Institute of Human Virology, University of Maryland School of Medicine, Baltimore, MD, USA
54 Summerland Research and Development Centre, Agriculture and Agri-Food Canada, Summerland, BC, Canada

55 Department of Planning and Coordination, National Agriculture and Food Research Organization, Tsukuba, Japan

56 Asian Center for Bioresources and Environmental Sciences, University of Tokyo, Tokyo, Japan

57 School of Cellular and Molecular Medicine, University of Bristol, Bristol, UK

58 Department of Microbiology, Immunology and Pathology, Colorado State University, Fort Collins, CO, USA

59 International Crops Research Institute for the Semi-Arid Tropics (ICRISAT), Hyderabad, Telangana, India

60 Department of Virology, Helsinki University Hospital, University of Helsinki, Helsinki, Finland

61 School of Biological Sciences, University of Queensland, St. Lucia, QLD, Australia

62 Fujian Province Key Laboratory of Plant Virology, Institute of Plant Virology, Fujian Agriculture and Forestry University, Fuzhou, Fujian, China

63 University of Nebraska Medical Center, Omaha, NE, USA

64 Global Viral, San Francisco, CA, USA

65 Horticultural Crop (Fruit Trees) Biology and Germplasm Creation, Ministry of Agriculture, Wuhan, Hubei, China

66 Key Laboratory of Plant Pathology of Hubei Province, Wuhan, Hubei, China

67 College of Plant Science and Technology, Huazhong Agricultural University, Wuhan, Hubei, China

68 State Key Laboratory for Molecular Virology and Genetic Engineering, Beijing, China

69 National Chung-Hsing University, Taichung City, Taiwan

70 Department of Zoonoses, State Key Laboratory for Infectious Disease Prevention and Control, Collaborative Innovation Center for Diagnosis and Treatment of Infectious Diseases, National Institute for Communicable Disease Control and Prevention, Chinese Center for Disease Control and Prevention, Changping, Beijing, China

71 State Key Laboratory for Biology of Plant Diseases and Insect Pests, Institute of Plant Protection, Chinese Academy of Agricultural Sciences, Beijing, China

72 Division of Clinical Research (DCR), Integrated Research Facility at Fort Detrick (IRF-Frederick), National Institute of Allergy and Infectious Diseases (NIAID), National Institutes of Health (NIH), B-8200 Research Plaza, Fort Detrick, Frederick, MD 21702, USA 